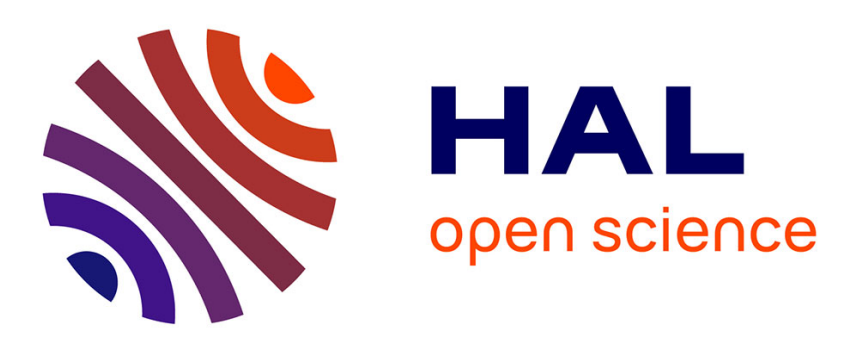

\title{
Discrete Cuckoo Search with Local Search for Max-cut Problem
}

\author{
Yingying $\mathrm{Xu}$, Zhihua Cui, Lifang Wang
}

\section{To cite this version:}

Yingying Xu, Zhihua Cui, Lifang Wang. Discrete Cuckoo Search with Local Search for Max-cut Problem. 2nd International Conference on Intelligence Science (ICIS), Oct 2017, Shanghai, China. pp.66-74, 10.1007/978-3-319-68121-4_7 . hal-01820945

\section{HAL Id: hal-01820945 \\ https://hal.inria.fr/hal-01820945}

Submitted on 22 Jun 2018

HAL is a multi-disciplinary open access archive for the deposit and dissemination of scientific research documents, whether they are published or not. The documents may come from teaching and research institutions in France or abroad, or from public or private research centers.
L'archive ouverte pluridisciplinaire HAL, est destinée au dépôt et à la diffusion de documents scientifiques de niveau recherche, publiés ou non, émanant des établissements d'enseignement et de recherche français ou étrangers, des laboratoires publics ou privés. 


\title{
Discrete Cuckoo Search with Local Search for Max-cut Problem
}

\author{
Yingying Xu, Zhihua Cui ${ }^{(\bowtie)}$, Lifang Wang \\ Complex System and Computational Intelligence Laboratory, \\ Taiyuan University of Science and Technology, Taiyuan, Shanxi, 030024, China.$$
\begin{gathered}
\text { s_yingyingxud163.com } \\
\text { zhihua.cuidhotmail.com } \\
\text { wlf1001@163.com }
\end{gathered}
$$

\begin{abstract}
Max-cut problem is a well-known NP-hard problem, in this paper, a hybrid meta-heuristic algorithm is designed to solve it. In this algorithm, the discrete cuckoo search is employed to find the approximate satisfied solution, while the local optimal solution is used to further improve the performance. To test the validity, three other algorithms are used to compare, simulation results show our modification is effective.
\end{abstract}

Keywords: Max-cut problem, discrete cuckoo search algorithm, local search strategy.

\section{Introduction}

Max-cut problem is a well-known NP-hard graph problem. For a graph $G=(V, E), V=\{1,2, \ldots, \mathrm{n}\}$ is vertex set and $E$ is the ordered set of undirected edges. Let $w_{i j}$ be the weight associated with edge $\{i, j\} \in E$, then max-cut problem is to find an optimal partition $\left(V_{1}, V_{2}\right)\left(V_{1} \cap V_{2}=\phi, V_{1} \cup V_{2}=V\right)$, so that the total weights of the edges crossing different subsets is maximized, in other words, the objective function can be represented as:

$$
\sum_{i \in V_{1}, j \in V_{2}} w_{i j}=\sum_{i<j} w_{i j} \cdot \frac{1-x_{i} x_{j}}{2}
$$

where $x_{i} \in\{1,-1\}(i=1,2, \cdots, n), x_{i}=1$ represents $x_{i} \in V_{1}$, as well as $x_{i}=-1$ denotes $x_{i} \in V_{2}$.

During the past years, many algorithms have been designed to solve it, including exact algorithm, approximate algorithm and heuristic algorithm [1-2]. Heuristic algorithm [3-5] is an umbrella for all population-based stochastic optimization algorithm inspired by heuristic information [6], such as ant colony optimization [7-8], fruit fly 
optimization [9], particle swarm optimization [10-12], artificial bee colony [13-16], social emotional optimisation algorithm [17], firefly algorithm [18-22] and bat algorithm [23-25].

For max-cut problem, Laguna et al. [26] designed a hybrid version of cross entropy method, while Lin [27] proposed a discrete dynamic convexized method. Festa et al. [28] investigated several heuristics derived from greedy randomized adaptive search procedure and variable neighborhood search. In 2007, Wang [29] proposed a hybrid algorithm combining with chaotic discrete Hopfield neural network and genetic particle swarm optimization, while in 2011, Wang [30] designed another combination with tabu Hopfield neural network and estimation of distribution algorithm. Inspired by this work, Lin [31] designed an integrated method combined with particle swarm optimization and estimation of distribution algorithm, and a local search strategy is employed to improve the accuracy. Shylo [32] also employed global equilibrium search algorithms and tabu search to improve the accuracy.

In this paper, we propose a new heuristic algorithm to combining the cuckoo search algorithm and local search strategy, and apply it to solve max-cut problem. The rest of this paper is organized as follows: In Section 2, the details of our proposed hybrid algorithm are presented, as well as the simulation results are reported in Section 3.

\section{Hybrid Algorithm}

\subsection{Discrete Cuckoo Search Algorithm}

Cuckoo search algorithm was proposed in 2009 [33], up to now, many variants are proposed to improve the performance [34-36]. However, max-cut problem is a combination problem, and a discrete cuckoo search algorithm is designed to solve it.

In this discrete version, the position movement will use the following strategies [37]:

Strategy 1:

$$
x_{i j k}^{m+1}=\left\{\begin{array}{c}
x_{i j k}^{m}, \text { rand }() \leq \operatorname{Sig}(\text { Step }) \\
-1, \text { other }
\end{array}\right.
$$

where

$$
\operatorname{Sig}(\text { Step })=1 /(1+\exp (- \text { Step }))
$$

Strategy 2:

$$
x_{i j k}^{m+1}=\left\{\begin{array}{c}
-1, \operatorname{rand}() \leq \operatorname{Sig}(\text { Step }) \\
x_{i j k}^{m}, \text { other }
\end{array}\right.
$$

where

$$
\operatorname{Sig}(\text { Step })=1-2 /(1+\exp (- \text { Step }))
$$

Strategy 3: 


$$
x_{i j k}^{m+1}=\left\{\begin{array}{c}
1, \operatorname{rand}() \leq \operatorname{Sig}(\text { Step }) \\
x_{i j k}^{m}, \text { other }
\end{array}\right.
$$

where

$$
\operatorname{Sig}(\text { Step })=2 /(1+\exp (- \text { Step }))-1
$$

The most difference among three strategy is the sigma function sig (step), and jump path step is a random number with Levy distribution, to provide a deep insight, the Eq. (3), Eq.(5) and Eq.(7) are plotted in Fig.1-Fig.3.

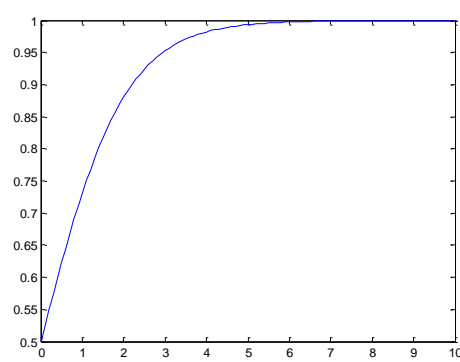

Fig. 1. Illustration for Eq.(3)

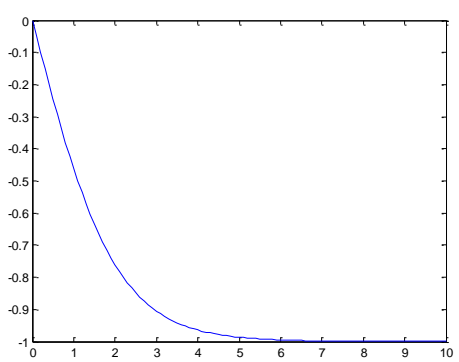

Fig. 2. Illustration for Eq.(5)

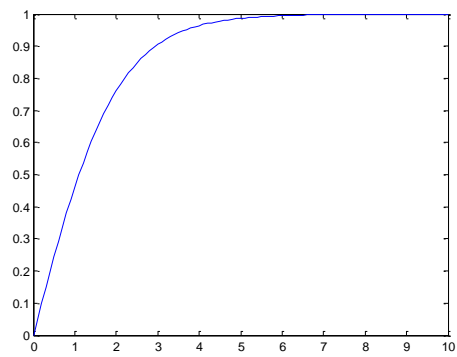

Fig.3. Illustration for Eq.(7)

Algorithm 1 is the pseudocode of our discrete cuckoo search algorithm, where $p_{r}$ is the predefined factor, and $P_{a}$ is the probability of being discovered by the host bird.

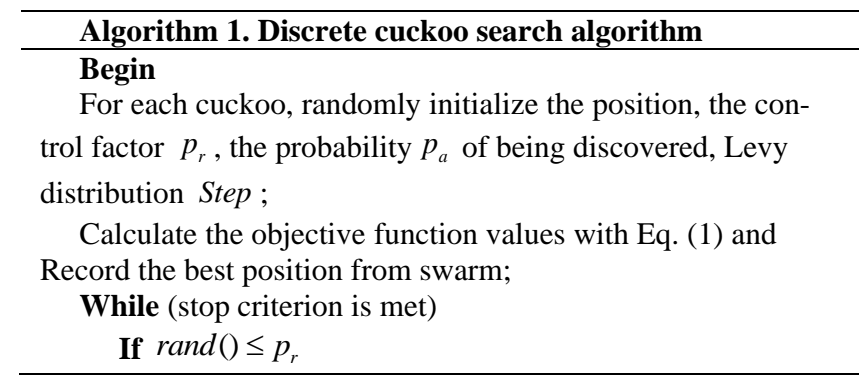




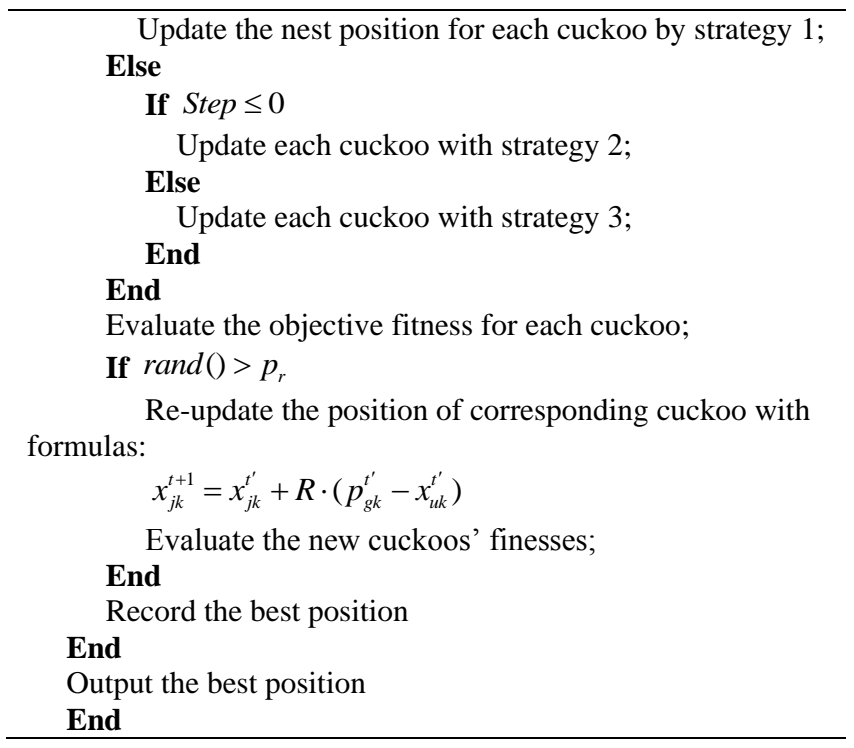

\subsection{Local Search Strategy}

For any partition $\left(V_{1}, V_{2}\right)$, if the vertex $j$ is moved from the current partition to another subset, the gain index $g_{j}$ is defined as follows:

$$
g_{j}=\left\{\begin{array}{l}
\sum_{\{j, k\} \in E, k \in V_{1}} w_{j k}-\sum_{\{j, k\} \in E, k \in V_{2}} w_{j k}, j \in V_{1} \\
\sum_{\{j, k\} \in E, k \in V_{2}} w_{j k}-\sum_{\{j, k\} \in E, k \in V_{1}} w_{j k}, j \in V_{2}
\end{array}\right.
$$

Gain $g_{j}>0$ means the vertex $j$ should be moved with a lower objective function. With this manner, the following local search strategy is introduced:

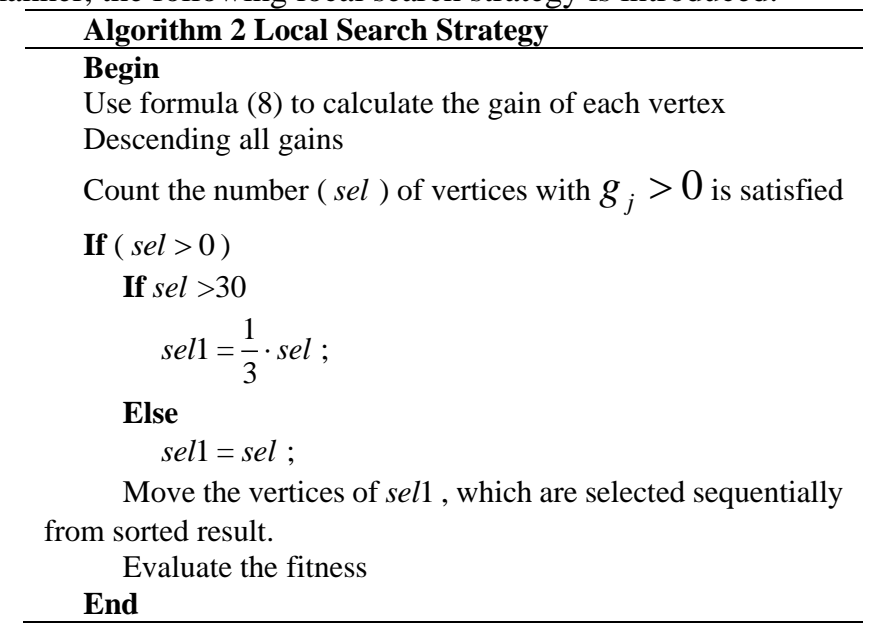




\section{End}

\subsection{Proposed Hybrid Algorithm}

Our modification is a hybrid meta-heuristic method combining a discrete cuckoo search algorithm and local search strategy. The discrete cuckoo search is employed to find the approximate satisfied solution, while the local optimal solution is used to further improve the performance for the obtained approximate satisfied solution. Furthermore, to avoid the premature convergence, a mutation strategy is also employed to avoid the premature convergence. The pseudocode of our hybrid algorithm is listed in Algorithm 3. For each cuckoo, the mutation operation will randomly take $0.1 \%$ vertices to flip. We find that the algorithm will be improved after adding the mutation operation.

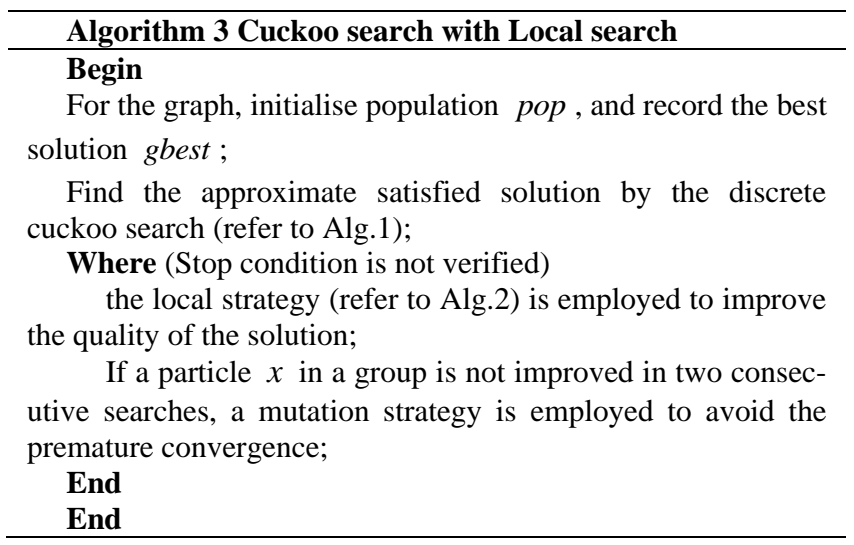

\section{Performance Evolution}

To test the performance of our proposed hybrid algorithm, G-set graph benchmarks are employed, and compared with the following algorithms:

- Hybridizing the cross-entropy method (HCE, in briefly) [26];

- A new lagrangian net algorithm (LNA, in briefly) [38];

- Discrete Hopfield network with estimation of distribution algorithm (DHNNEDA, in briefly) [14]

- $\quad$ Discrete cuckoo search with local search (DCSLS, in briefly)

The program is implemented with MATLAB. In this set of instances, the number of vertex range from 800 to 3000 . the control factor $p_{r}$ is 0.3 , the probability $p_{a}$ are both set to 0.5 , the total generation is 500 .

For chosen benchmarks, each instance will run 50 times, Table 1 provides the optimal value achieved by the three algorithms and our proposed algorithm. The last line noted as $" w / t / l$ " is the comparison results between our proposed DCSLS and its competitors. " $w / t / l$ " represents our algorithm wins in $w$ functions, ties in $t$ functions, and 
loses in $l$ functions. It means DCSLS is better than HCE for six functions, while only worse than HCE for three functions. DCSLS are superior than DHNN-EDA and LNA for six functions too, while DHNN-EDA and LNA only better than DCSLS with two and three functions, respectively. In one word, DCSLS achieves the best performance when compared with HCE, DHNN-EDA and LNA.

Table 1: Comparison of the results

\begin{tabular}{cccccc}
\hline $\begin{array}{c}\text { In- } \\
\text { stances }\end{array}$ & best & HCE & $\begin{array}{c}\text { DHNN- } \\
\text { EDA }\end{array}$ & LNA & DCSLS \\
\hline G1 & 11624 & 11584 & 11614 & 11490 & 11607 \\
G2 & 11620 & 11595 & 11599 & 11505 & 11599 \\
G3 & 11622 & 11574 & 11617 & 11511 & 11605 \\
G11 & 564 & 552 & 494 & 560 & 530 \\
G12 & 556 & 542 & 476 & 546 & 528 \\
G13 & 582 & 564 & 520 & 572 & 552 \\
G14 & 3064 & 3030 & 3027 & 3023 & 3035 \\
G15 & 3050 & 3012 & 2988 & 2996 & 3016 \\
G16 & 3052 & 3015 & 3001 & 2994 & 3018 \\
$w / t / l$ & & $\mathbf{6 / 0 / 3}$ & $\mathbf{6 / 1 / 2}$ & $\mathbf{6 / 0 / 3}$ & \\
\hline
\end{tabular}

To provide a deep comparison, two non-parametric statistics tests: Friedman test and Wilcoxon test, are employed to show the differences among these four algorithms. In Tab.2, the ranking value is: DCSLS $<\mathrm{HCE}<$ DHNN-EDA $<$ LNA, DCSLS maintains the lowest ranking, it means the performance of DCSLS is more better. Tab. 3 implies there is significantly difference between DCSLS and DHNN-EDA.

Table 2: Friedman test

\begin{tabular}{cc}
\hline & $\begin{array}{c}\text { Rank- } \\
\text { Value }\end{array}$ \\
\hline HCE & 2.33 \\
DCSLS & 1.94 \\
LNA & 2.89 \\
DHNN-EDA & 2.83 \\
\hline
\end{tabular}

Table 3: Wilcoxon test

\begin{tabular}{cc}
\hline DCSLS vs & P-Value \\
\hline HCE & 0.594 \\
LNA & 0.192 \\
DHNN-EDA & 0.050 \\
\hline
\end{tabular}

\section{Conclusion}

In this paper, a new hybrid algorithm combining with discrete cuckoo search and local search strategy is designed. The cuckoo update manner of discrete cuckoo search is the same as [37], while the local search strategy is designed. Simulation results show our modification achieves the best performance when compared with other three algorithms.

Acknowledgments. This research is supported by the Natural Science Foundation of Shanxi Province under No. 201601D011045. 


\section{References}

1. Xia, Y., Xu, Z.: An efficient Lagrangian smoothing heuristic for Max-Cut. Indian Journal of Pure and Applied Mathematics,41(5), 683-700 (2010)

2. Marti, R., Duarte, A., Laguna, M.: Advanced Scatter Search for the Max-Cut Problem. INFORMS J. Comput. 21(1), 26-38(2009)

3. Wang, G.G., Deb, S., Gao, X.Z., Coelho, L.: A new metaheuristic optimization algorithm motivated by elephant herding behavior. Int. J. Bio-Inspired Comput. 8(6), 394-409 (2016)

4. Bilbao, M.N., Ser, J.D., Salcedo-Sanz, S., Casanova-Mateo, C.: On the application of multiobjective harmony search heuristics to the predictive deployment of firefighting aircrafts: a realistic case study. Int. J. Bio-Inspired Comput. 7(5), 270-284 (2015)

5. Rajakumar, R., Dhavachelvan, P., Vengattaraman, T.: A survey on nature inspired metaheuristic algorithms with its domain specifications, In: International Conference on Communication and Electronics Systems, pp.550-555 (2016)

6. Xiao, R., Zhang, Y., Huang, Z.: Emergent computation of complex systems: a comprehensive review. Int. J. Bio-Inspired Comput. 7(2), 75-97 (2015)

7. Dorigo, M., Gambardella, L.M., Middendorf, M., Stutzle, T.: Special section on ant colony optimization. IEEE Trans. Evol. Comput. 6(4), 317-320 (2002)

8. Stodola, P., Mazal, J.: Applying the ant colony optimisation algorithm to the capacitated multi-depot vehicle routing problem. Int. J. Bio-Inspired Comput. 8(4), 228-233 (2016)

9. Zhang, Y.W., Wu, J.T., Guo, X., Li,G.N.: Optimising web service composition based on differential fruit fly optimisation algorithm. Int. J. Comput. Sci. Math. 7(1), 87-101 (2016)

10. Eberhart, R.C., Shi, Y.H.: Special issue on particle swarm optimization. IEEE Trans. Evol. Comput. 8(3), 201-203 (2004)

11. Adewumi, A.O., Arasomwan, M.A.: On the performance of particle swarm optimisation with(out) some control parameters for global optimisation. Int. J. Bio-Inspired Comput. 8(1), 14-32 (2016)

12. Grillo, H., Peidro, D., Alemany, M., Mula, J.: Application of particle swarm optimisation with backward calculation to solve a fuzzy multi-objective supply chain master planning model. Int. J. Bio-Inspired Comput. 7(3), 157-169 (2015)

13. Lv, L., Wu, L.Y., Zhao, J., Wang, H., Wu, R.X., Fan, T.H., Hu, M., Xie, Z.F.: Improved multi-strategy artificial bee colony algorithm. Int. J. Comput. Sci. Math. 7(5), 467-475 (2016)

14. Sun, H., Wang, K., Zhao, J., Yu, X.: Artificial bee colony algorithm with improved special centre. Int. J. Comput. Sci. Math. 7(6), 548-553 ( 2016)

15. Lu, Y., Li, R.X., Li, S.M.: Artificial bee colony with bidirectional search. Int. J. Comput. Sci. Math. 7(6), 586-593 (2016)

16. Yu, G.: A new multi-population-based artificial bee colony for numerical optimization. Int. J. Comput. Sci. Math. 7(6), 509-515 (2016)

17. Guo, Z.L., Wang, S.W., Yue, X.Z., Yin, B.Y., Deng, C.S., Wu, Z.J.: Enhanced social emotional optimisation algorithm with elite multi-parent crossover. Int. J. Comput. Sci. Math. 7(6), 568-574 (2016)

18. Wang, H., Wang, W.J., Zhou, X.Y.: Firefly algorithm with neighborhood attraction. Inf. Sci.382, 374-387 (2017)

19. Wang, H., Wang, W.J., Sun, H.: Firefly algorithm with random attraction. Int. J. Bio-Inspired Comput. 8(1), 33-41 (2016)

20. Yu, G.: An improved firefly algorithm based on probabilistic attraction. Int. J. Comput. Sci. Math. 7(6), 530-536 (2016) 
21. Nasiri, B., Meybodi, M.R.: History-driven firefly algorithm for optimisation in dynamic and uncertain environments. Int J of Bio-Inspired Computation 8(5):326-339 (2016)

22. Fister, I., Fister, I., Yang, X.S., Brest, J.: A comprehensive review of firefly algorithms. Swarm Evol. Comput.13, 34-46 (2013)

23. Yang, X.S., Gandomi, A.H.: Bat algorithm: a novel approach for global engineering optimization. Eng. Comput. 29(5-6), 464-483 (2012)

24. Cai, X., Gao, X.Z., Xue, Y.: Improved bat algorithm with optimal forage strategy and random disturbance strategy. Int J of Bio-Inspired Computation 8(4):205-214 (2016)

25. Xue, F., Cai, Y., Cao, Y., Cui, Z., Li, F. Optimal parameter settings for bat algorithm. Int J of Bio-Inspired Computation 7(2):125-128 (2015)

26. Laguna, M., Duarte, A., Marti, R.: Hybridizing the cross-entropy method: an application to the max-cut problem. Comput. Oper. Res. 36(2), 487-498 (2009)

27. Lin, G., Zhu, W.: A discrete dynamic convexized method for the max-cut problem. Ann. Oper. Res. 196(1), 371-390 (2012).

28. Festa, P., Pardalos, P.M., Resende, M.G.C., Ribeiro, C.C.: Randomized heuristics for the MAX-CUT problem. Optim. Method Softw. 17(6), 1033-1058(2002).

29. Wang, J.: A Memetic Algorithm with Genetic Particle Swarm Optimization and Neural Network for Maximum Cut Problems. In: International Conference on Life System Modeling and Simulation, pp.297-306 (2007)

30. Wang, J., Zhou, Y., Yin, J.: Combining tabu Hopfield network and estimation of distribution for unconstrained binary quadratic programming problem. Expert Syst. Appl. 38(12), 14870-14881 (2011)

31. Lin, G., Guan, J.: An Integrated Method Based on PSO and EDA for the Max-Cut Problem. Comput. Intell. Neurosci., DOI:10.1155/2016/3420671 (2016)

32. Shylo, V.P., Shylo, O.V.: Solving the maxcut problem by the global equilibrium search. Cybern. Syst. Anal. 46(5), 744-754(2010)

33. Yang, X.S., Deb, S.: Cuckoo search via Levy flights, In: World Congress on Nature \& Biologically Inspired Computing, pp.210-214 (2009)

34. Cui, Z.H., Sun, B., Wang, G.G., Xue, Y.: A novel oriented cuckoo search algorithm to improve DV-hop performance for cyber-physical systems. J. Parallel Distrib. Comput. 103,42$52(2017)$

35. Zhang, M.Q., Wang, H., Cui, Z.H., Chen, J.J.: Hybrid multi-objective cuckoo search with dynamical local search. Memetic Comp. DOI:10.1007/s12293-017-0237-2 (2017)

36. Li, F.X., Cui, Z.H., Sun, B.: DV-hop localisation algorithm with DDICS. Int. J. Comput. Sci. Math. 7(3), 254-262 (2016)

37. Feng, D.K., Ruan, Q., Du, L.M.: Binary cuckoo search algorithm. Journal of Computer Applications 33(6), 1566-1570 (2013) (in Chinese)

38. Xu, F.M., Ma, X.S., Chen, B.L.: A new lagrangian net algorithm for solving max-bisection problems. J. Comput. Appl. Math. 235(13), 3718-3723(2011). 